Preprint from

UMN-TH-1211/93

University of Minnesota

August 6, 1993

\title{
Spontaneously Broken Lorentz Invariance in Three-Dimensional Gauge Theories
}

\author{
YUTAKA HOSOTANI \\ School of Physics and Astronomy, University of Minnesota \\ Minneapolis, MN 55455, U.S.A. \\ Type-set by plain $T_{E} X$
}

\begin{abstract}
In a wide class of three-dimensional Abelian gauge theories with a bare Chern-Simons term, the Lorentz invariance is spontaneously broken by dynamical generation of a nonvanishing magnetic field. A detailed computation of an energy density of the true vacuum is given. The originally massive photon becomes massless, fulfilling the role of a NambuGoldstone boson associated with the spontaneous breaking of the Lorentz invariance.
\end{abstract}

Gauge theory in (2+1)-dimensions is rich. A decade ago it was shown that in a general QED model a Chern-Simons term is generated by radiative corrections so that a photon becomes topologically massive. ${ }^{1}$ It also has been argued, by both analytical and numerical methods, that in a T-symmetric QED chiral symmetry is dynamically broken. ${ }^{2,3}$

In this paper we would like to show that in a wide class of Abelian gauge theories with a bare Chern-Simons term it is energetically favored to generate a magnetic field, thus the Lorentz invariance being spontaneously broken. The true vacuum is a Hall liquid of fermions. A photon becomes massless, fulfilling the role of a Nambu-Goldstone boson. The model is important in describing electron systems on a two-dimensional lattice near half filling. ${ }^{4}$ The mechanism of dynamical generation of a magnetic field is important in non-relativistic context as well, particularly in the fractional quantum Hall effect. ${ }^{5}$

Consider

$$
\begin{aligned}
\mathcal{L}= & -\frac{1}{4} F_{\mu \nu} F^{\mu \nu}-\frac{\kappa}{2} \varepsilon^{\mu \nu \rho} A_{\mu} \partial_{\nu} A_{\rho} \\
& +\sum_{a} \frac{1}{2}\left[\bar{\psi}_{a},\left(\gamma_{a}^{\mu}\left(i \partial_{\mu}+q_{a} A_{\mu}\right)-m_{a}\right) \psi_{a}\right] .
\end{aligned}
$$

Here $\psi_{a}$ is a two-component Dirac spinor. In $(2+1)$-dimensions there are two kinds of spinors, corresponding to the signature of two-dimensional Dirac matrices

$$
\eta_{a}=\frac{i}{2} \operatorname{Tr} \gamma_{a}^{0} \gamma_{a}^{1} \gamma_{a}^{2}= \pm 1
$$


We shall take a representation $\gamma_{a}^{\mu}=\left(\eta_{a} \sigma_{3}, i \sigma_{1}, i \sigma_{2}\right)$ where $\sigma_{j}$ 's are Pauli matrices. The model is invariant under charge conjugation. Further, the transformation $m_{a} \rightarrow-m_{a}$ is equivalent to the transformation $\gamma_{a}^{\mu} \rightarrow-\gamma_{a}^{\mu}$ or $\eta_{a} \rightarrow-\eta_{a}$. Hence one can take $q_{a}>0$ and $m_{a}>0$ without loss of generality.

Suppose that a magnetic field $B$ is spontaneously generated. We split $A_{\mu}=A_{\mu}^{(0)}+A_{\mu}^{(1)}$ where $A^{\mu(0)}=-B x_{2} \delta^{\mu 1}$. The Dirac equation in the presence of a constant magnetic field is exactly solved. ${ }^{6}$ Energy eigenvalues are

$$
E^{2}=\omega_{n}^{2} \quad, \quad \omega_{n}=\sqrt{m^{2}+\frac{2 n}{l^{2}}} \quad(n=0,1,2, \cdots)
$$

where $l$ is the magnetic length: $1 / l^{2}=|q B|$. We have suppressed the species index $a$.

There is asymmetry in the lowest Landau level $(n=0)$. Depending on the signs of $\eta$ and $q B$, either positive or negative energy states exsit. For instance, for $\eta=+$ and $q B>0$, eigenfunctions are

$$
\begin{aligned}
& u_{0 p}=\frac{1}{\left(l L_{1}\right)^{1 / 2}} e^{-i \omega_{0} t-i k x_{1}}\left[\begin{array}{c}
v_{0}(\xi) \\
0
\end{array}\right] \\
& u_{n p}=\frac{1}{\left(l L_{1}\right)^{1 / 2}} e^{-i \omega_{n} t-i k x_{1}} \frac{1}{\sqrt{2 \omega_{n}}}\left[\begin{array}{c}
\sqrt{\omega_{n}+m} v_{n}(\xi) \\
-i \sqrt{\omega_{n}-m} v_{n-1}(\xi)
\end{array}\right] \quad(n \geq 1) \\
& w_{n p}=\frac{1}{\left(l L_{1}\right)^{1 / 2}} e^{+i \omega_{n} t-i k x_{1}} \frac{1}{\sqrt{2 \omega_{n}}}\left[\begin{array}{c}
\sqrt{\omega_{n}-m} v_{n}(\xi) \\
+i \sqrt{\omega_{n}+m} v_{n-1}(\xi)
\end{array}\right] \quad(n \geq 1)
\end{aligned}
$$

Here $k=2 \pi p / L_{1}$ and $\xi=\left(x_{2} / l\right)-k l$ where $p$ is an integer. $v_{n}(z)=(-1)^{n} 2^{-n / 2} \pi^{-1 / 4}(n !)^{-1 / 2}$ $e^{z^{2} / 2}\left(d^{n} e^{-z^{2}} / d z^{n}\right)$ is the $n$-th eigenfunction in the harmonic oscillator problem. We have adopted a periodic boundary condition in the $x_{1}$-direction, understanding the $L_{1} \rightarrow \infty$ limit at the end. The spatial part of the wave function of the $n=0$ mode is independent of mass $m .^{7}$

Solutions for $q B<0$ are obtained by charge conjugation, namely by $\left\{u^{c}(x)=U_{c} \bar{u}(x)^{t}\right.$, $\left.w^{c}(x)\right\}$ where $U_{c}=\gamma^{2}$. Solutions for $\eta=-$ are obtained by the transformation $t \rightarrow-t$ from the corresponding ones in the $\eta=+$ case.

In the expansion of $\psi(x)$ one associates annihilation (creation) operators for positive (negative) energy solutions. For instance, for $\eta=+$,

$$
\psi(x)=\sum_{n p} a_{n p}\left\{\begin{array}{l}
u_{n p}(x) \\
w_{n p}^{c}(x)
\end{array}\right\}+\sum_{n p} b_{n p}^{\dagger}\left\{\begin{array}{l}
w_{n p}(x) \\
u_{n p}^{c}(x)
\end{array}\right\} \quad \text { for }\left\{\begin{array}{l}
q B>0 \\
q B<0
\end{array}\right.
$$

with normal anticommutation relations among $\left\{a_{n p}, b_{n p}\right\}$. The summation over $n$ runs from 0 or 1 for the $u_{n p}$ or $w_{n p}$ term, respectively.

In the massless limit the perturbative ground state with $B \neq 0$ is infinitely degenerate. As $\omega_{0}=0$, it costs no energy to put a fermion in the lowest Landau level. The degeneracy is lifted by radiative corrections. In this paper we consider two typical states, for sufficiently small masses, with the lowest Landau level in each speceis being empty $(\nu=0)$ or completely filled $(\nu=1)$. The case of fractional filling will be examined in a separate paper. It will be found that a state of the mixture of $\nu=0$ and $\nu=1$ among various speceis has a lower energy than the perturbative vaccum with $B=0$. 
The charge $Q=\int d^{2} x \frac{1}{2} q\left[\psi^{\dagger}, \psi\right]$ is found, by inserting (5), to be

$$
Q=q \sum_{p}\left\{\begin{array}{c}
a_{0 p}^{\dagger} a_{0 p}-\frac{1}{2} \\
-b_{0 p}^{\dagger} b_{0 p}+\frac{1}{2}
\end{array}\right\}+q \sum_{n=1}^{\infty} \sum_{p}\left(a_{n p}^{\dagger} a_{n p}-b_{n p}^{\dagger} b_{n p}\right) .
$$

Here the upper and lower components correspond to $\eta \epsilon(B)>0$ and $<0$, respectively.

The time component of $\partial_{\nu} F^{\nu \mu}-\kappa \varepsilon^{\mu \nu \rho} \partial_{\nu} A_{\rho}=j^{\mu}$ and the relation (6) imply that

$$
\kappa B=\left\langle j^{0}\right\rangle=\sum_{a} \eta_{a} \epsilon(B) q_{a}\left(\nu_{a}-\frac{1}{2}\right) \cdot \frac{q_{a}|B|}{2 \pi} .
$$

Hence for $B \neq 0$

$$
\kappa=\frac{1}{2 \pi} \sum_{a} \eta_{a} q_{a}^{2}\left(\nu_{a}-\frac{1}{2}\right)
$$

This necessary condition, as is shown below, is related to the Nambu-Goldstone theorem. The value of $B$ is to be determined by minimizing the energy density.

We need to compare the energy density of the perturbative vacuum with $B=0, \mathcal{E}_{0}(e)$, with that of the non-perturbative vacuum, $\mathcal{E}\left(e ; q_{a} B, \nu_{a}\right)$. Here we have collectively characterised the interaction coupling $A_{\mu}^{(1)} j^{\mu}$ by $e$, supposing that all $q_{a}$ 's in the fluctuation part are proportional to $e$. Fermions couple to a dynamically generated magnetic field in the combination $q_{a} B$ so that the energy density in the nonperturbative vacuum may be regarded as a function of $e,\left\{q_{a} B\right\}$, and filling factors $\left\{\nu_{a}\right\}$.

It is beneficial to compare these energy densities with the corresponding ones when the fluctuation coupling $e$ is turned off, while $\left\{q_{a} B\right\}$ kept fixed. We write

$$
\begin{aligned}
\Delta \mathcal{E} & =\mathcal{E}\left(e ; q_{a} B, \nu_{a}\right)-\mathcal{E}_{0}(e) \\
& =\left\{\mathcal{E}\left(e ; q_{a} B, \nu_{a}\right)-\mathcal{E}\left(0 ; q_{a} B, \nu_{a}\right)\right\}-\left\{\mathcal{E}_{0}(e)-\mathcal{E}_{0}(0)\right\} \\
& +\left\{\mathcal{E}\left(0 ; q_{a} B, \nu_{a}\right)-\mathcal{E}_{0}(0)\right\} \\
& \equiv \Delta \mathcal{E}^{(1)}-\Delta \mathcal{E}^{(2)}+\Delta \mathcal{E}^{(3)}
\end{aligned}
$$

$\Delta \mathcal{E}^{(3)}$ is the difference in energy densities in the "free" $(e=0)$ theories. It is easy to see

$$
\Delta \mathcal{E}^{(3)}=\frac{1}{2} B^{2}+\sum_{a} \frac{1}{2^{5 / 2} \pi^{2}} \zeta\left(\frac{3}{2}\right)\left|q_{a} B\right|^{3 / 2}
$$

in the massless fermion limit. The first term is the Maxwell energy, whereas the second comes from the shift in zero point energies of fermions. $\Delta \mathcal{E}^{(3)}$ is always minimized at $B=0$.

$\Delta \mathcal{E}^{(1)}$ and $\Delta \mathcal{E}^{(2)}$ represent the shift in energy densities when the fluctuation interaction $A_{\mu}^{(1)} j^{\mu}$ is turned on. We employ an exact formula ${ }^{8}$

$$
\Delta \mathcal{E}^{(1)}=i \int_{0}^{e} \frac{d e}{e} \int \frac{d^{3} p}{(2 \pi)^{3}} \operatorname{tr} D_{0}^{-1}(p)\left\{D\left(p ; q_{a} B, \nu_{a}\right)-D_{0}(p)\right\}
$$

Here $D_{0}^{\mu \nu}(p)$ and $D^{\mu \nu}\left(p ; q_{a} B, \nu_{a}\right)$ are the bare and full photon propagators in the nonperturbative vacuum. The trace is taken over Lorentz indices. For $\Delta \mathcal{E}^{(2)}, D\left(p ; q_{a} B, \nu_{a}\right)$ is replaced by $D(p ; B=0)$. $D_{0}(p)$, independent of $B$, is given by

$$
D_{0}^{\mu \nu}(p)=\frac{1}{p^{2}-\kappa^{2}}\left\{g^{\mu \nu}-\kappa^{2} \frac{p^{\mu} p^{\nu}}{\left(p^{2}\right)^{2}}-i \kappa \varepsilon^{\mu \nu \rho} \frac{p_{\rho}}{p^{2}}\right\} .
$$


$D$ is related to one-particle irreducible diagrams, $\Gamma$, by $D^{-1}=D_{0}^{-1}-\Gamma$. The rotational invariance and current conservation imply that ${ }^{9}$

$$
\begin{aligned}
& \Gamma^{00}=\vec{p}^{2} \Pi_{0} \\
& \Gamma^{0 j}=p_{0} p_{j} \Pi_{0}-i \epsilon^{j k} p_{k} \Pi_{1} \\
& \Gamma^{j 0}=p_{0} p_{j} \Pi_{0}+i \epsilon^{j k} p_{k} \Pi_{1} \\
& \Gamma^{j k}=\delta^{j k} p_{0}^{2} \Pi_{0}+i \epsilon^{j k} p_{0} \Pi_{1}-\left(\vec{p}^{2} \delta^{j k}-p_{j} p_{k}\right) \Pi_{2}
\end{aligned}
$$

All $\Pi_{j}$ 's are functions of $\left|p_{0}\right|$ and $|\vec{p}|$. (Here $p_{j} \equiv p^{j}$ )

If $\Gamma(p)$ is approximated by $\mathrm{O}\left(e^{2}\right)$ diagrams, then the integration over $e$ in (11) can be trivially performed, yielding

$$
\begin{aligned}
\Delta \mathcal{E}^{(1)}-\Delta \mathcal{E}^{(2)} & =-\frac{i}{2} \int \frac{d^{3} p}{(2 \pi)^{3}}\left\{\left.\operatorname{tr} \ln \left(1-\Gamma^{(2)} D_{0}\right)\right|_{q B, \nu}-(B \rightarrow 0)\right\} \\
& =-\frac{i}{2} \int \frac{d^{3} p}{(2 \pi)^{3}} \ln \frac{\left(1+\Pi_{0}\right)\left\{1+\frac{1}{p^{2}}\left(p_{0}^{2} \Pi_{0}-\vec{p}^{2} \Pi_{2}\right)\right\}-\frac{1}{p^{2}}\left(\kappa-\Pi_{1}\right)^{2}}{(B \rightarrow 0)} .
\end{aligned}
$$

If $\Pi_{j}(p)$ is further approximated by $\Pi_{j}(0)$, then the above formula reduces to the one-loop effective action. However, we shall see below that it is absolutely necessary and crucial to take full account of the momentum dependence.

To find $\Pi_{j}$, we need a fermion propagator, $S(x, y)=-i\langle T[\psi(x) \bar{\psi}(y)]\rangle$, in the nonperturbative vacuum. First we note that

$$
\begin{aligned}
S(x, y)_{B>0} & =e^{-i\left(x_{1}-y_{1}\right)\left(x_{2}+y_{2}\right) / 2 l^{2}} \cdot S_{0}(x-y) \\
S_{0}(p)_{\eta=-} & =S_{0}\left(-p_{0}, \vec{p}\right)_{\eta=+} \\
S(x, y)_{B<0} & =U_{c} S(y, x)_{B>0}^{t} U_{c}^{\dagger}
\end{aligned}
$$

which follows from (4) and (5). It is straightforward to see ${ }^{10}$ that

$$
\begin{aligned}
& S_{0}(p)_{\nu=0}^{\eta=+}=-i l^{2} \int_{0}^{\infty} d t(\cos t)^{-1} e^{i\left(p_{0}^{2}-m^{2}+i \epsilon\right) l^{2} t-i \vec{p}^{2} l^{2} \tan t} \\
& \times\left[\begin{array}{cc}
\left(m+p_{0}\right) e^{i t} & -i\left(p_{1}-i p_{2}\right)(\cos t)^{-1} \\
-i\left(p_{1}+i p_{2}\right)(\cos t)^{-1} & \left(m-p_{0}\right) e^{-i t}
\end{array}\right] \\
& S_{0}(p)_{\nu=1}^{\eta=+}=S_{0}(p)_{\nu=0}^{\eta=+}+4 \pi i e^{-\vec{p}^{2} l^{2}} \delta\left(p_{0}-m\right)\left[\begin{array}{cc}
1 & 0 \\
0 & 0
\end{array}\right] .
\end{aligned}
$$

In the $B \rightarrow 0(l \rightarrow \infty)$ limit, $S_{0}(p)$ reduces to $(p \gamma-m+i \epsilon)^{-1}$.

$\Gamma^{(2) \mu \nu}(p)$ is given by

$$
\Gamma^{(2) \mu \nu}(p)=i q^{2} \int \frac{d^{3} k}{(2 \pi)^{3}} \operatorname{tr} \gamma^{\mu} S_{0}(k) \gamma^{\nu} S_{0}(k-p) .
$$

To $\mathrm{O}\left(e^{2}\right)$ a contribution from each fermion adds up to the total $\Pi_{j}$. An immediate consequence of (13), (15), and (17) is, for each fermion loop,

$$
\begin{aligned}
& \Pi_{j}^{\eta=-}= \pm \Pi_{j}^{\eta=+} \quad \text { for } \quad\left\{\begin{array}{c}
j=0,2 \\
j=1
\end{array}\right. \\
& \Pi_{j}^{B<0}=\Pi_{j}^{B>0} .
\end{aligned}
$$


The $\Pi_{0}$ and $\Pi_{2}$ parts of the integral (17) contain linear divergences, which need to be regularized. We employ the invariant Pauli-Villars regularization, i.e. for each fermion loop with either $\eta=+$ or - , we associate two regulator fields of $\eta=+$ and - , with equal weight, and let the regulator masses go to $\infty$ at the end. ${ }^{11}$ Divergent parts are independent of a mass $m$ and magnetic field $B$. Straightforward manipulations lead to

$$
\begin{aligned}
& {\left[\begin{array}{c}
\Pi_{0} \\
\Pi_{1} \\
p_{0}^{2} \Pi_{0}-\vec{p}^{2} \Pi_{2}
\end{array}\right]_{\eta=+, \nu=0}=\frac{q^{2} l e^{i \pi / 4}}{8 \pi^{3 / 2}} \int_{0}^{1} d w \int_{0}^{\infty} d s\left[\begin{array}{l}
f_{0} \\
f_{1} \\
f_{2}
\end{array}\right]} \\
& \quad \times \exp \left(\frac{i}{4}\left(1-w^{2}\right) s p_{0}^{2} l^{2}-\frac{i}{2} \frac{\cos w s-\cos s}{\sin s} \vec{p}^{2} l^{2}-i m^{2} l^{2} s-\epsilon s\right)
\end{aligned}
$$

where

$$
\begin{aligned}
& f_{0}=\frac{s^{1 / 2}}{\sin s}\left(1+\frac{\cos s}{\sin s} \frac{d}{d s}\right)(\cos w s-\cos s) \\
& f_{1}=-2 m \frac{s^{1 / 2}}{\sin s} \cos w s \\
& f_{2}=-s^{1 / 2} \frac{d}{d s}\left(\frac{\sin w s}{\sin s}\right) \cdot\left(w p_{0}^{2}-\frac{\sin w s}{\sin s} \vec{p}^{2}\right)+\frac{s^{1 / 2} \cos w s}{\sin s}\left(1-w^{2}\right) p_{0}^{2} \\
& \quad-\frac{s^{1 / 2}}{\sin s}\left(\frac{1}{\sin s}+\cos w s \frac{d}{d s}\right)\left(\frac{\cos w s-\cos s}{\sin s}\right) \cdot \vec{p}^{2}
\end{aligned}
$$

For $\nu=1$

$$
\begin{gathered}
{\left[\begin{array}{l}
\Pi_{0} \\
\Pi_{1} \\
\Pi_{2}
\end{array}\right]_{\substack{\eta=+\nu=1}}=\left[\begin{array}{l}
\Pi_{0} \\
\Pi_{1} \\
\Pi_{2}
\end{array}\right]_{\substack{\eta=+\nu=0}}+\frac{q^{2}}{\pi} \int_{0}^{\infty} d s\left[\begin{array}{c}
-\left(p_{0}\right)^{-1} \sin 2 m p_{0} l^{2} s \\
i \cos 2 m p_{0} l^{2} s \\
0
\end{array}\right]} \\
\times \exp \left(-2 i s+i p_{0}^{2} l^{2} s-\frac{\vec{p}^{2} l^{2}}{2}\left(1-e^{-2 i s}\right)-\epsilon s\right) .
\end{gathered}
$$

The induced Chern-Simons coefficient, $-\Pi_{1}$ at $p^{\mu}=0$, can be evaluated exactly.

$$
\begin{aligned}
& \left.\Pi_{1}(0)\right|_{B=0}=-\eta \frac{q^{2}}{4 \pi} \\
& \left.\Pi_{1}(0)\right|_{B \neq 0}=\eta\left(\nu-\frac{1}{2}\right) \frac{q^{2}}{2 \pi}
\end{aligned}
$$

The sign of $\Pi_{1}(0)$ flips when the lowest Landau level is filled $(\nu=1)$. We also note that $\left.\lim _{m \rightarrow 0} \Pi_{1}\left(p^{2} \neq 0\right)\right|_{B=0}=0$ so that two limits $m \rightarrow 0$ and $p \rightarrow 0$ do not commute with each other. The necessary condition (8) is expressed as

$$
\Pi_{1}(0)=\kappa,
$$

i.e. the bare Chern-Simons term must be exactly cancelled by the induced Chern-Simons term in order to have $B \neq 0$.

These functions are not analytic in $B$. The integrals in the $B \rightarrow 0(l \rightarrow \infty)$ limit are dominated at small $s$. Hence behavior at small $|B|$ is found by expanding $\cos s$ in the 
exponent and others in Taylor series in $s$. At $B=0$ (19) and (20) reproduce the standard result:

$$
\begin{aligned}
& \left.\Pi_{0}\right|_{B=0}=\left.\Pi_{2}\right|_{B=0}=\frac{q^{2}}{8 \pi} \frac{1}{\left(-p^{2}\right)^{1 / 2}}\left(\sqrt{z}+(1-z) \sin ^{-1} \frac{1}{\sqrt{1+z}}\right) \\
& \left.\Pi_{1}\right|_{B=0}=-\frac{q^{2}}{4 \pi} \sqrt{z} \sin ^{-1} \frac{1}{\sqrt{1+z}}
\end{aligned}
$$

where $z=4 m^{2} /\left(-p^{2}\right)$.

Recalling $q|B|=1 / l^{2}$, we see that

$$
\left.\Pi_{j}(p)\right|_{B, \nu=0}=\left.\Pi_{j}(p)\right|_{B=0}+\mathrm{O}\left(B^{2}\right) .
$$

When the lowest level is filled,

$$
\begin{aligned}
& \left.\Pi_{0}(p)\right|_{B, \nu=1}-\left.\Pi_{0}(p)\right|_{B=0} \sim \frac{q^{2}}{2 \pi} \frac{1}{p_{0}}\left\{\frac{1}{2-p^{2} l^{2}-2 m p_{0} l^{2}}-\frac{1}{2-p^{2} l^{2}+2 m p_{0} l^{2}}\right\} \\
& \left.\Pi_{1}(p)\right|_{B, \nu=1}-\left.\Pi_{1}(p)\right|_{B=0} \sim \eta \frac{q^{2}}{2 \pi}\left\{\frac{1}{2-p^{2} l^{2}-2 m p_{0} l^{2}}+\frac{1}{2-p^{2} l^{2}+2 m p_{0} l^{2}}\right\} .
\end{aligned}
$$

$\left.\Pi_{2}\right|_{\nu=1}=\left.\Pi_{2}\right|_{\nu=0}$ as noted above. For small $m$ a significant correction of $\mathrm{O}(|B|)$ arises only for $\Pi_{1}(p)$ at $\nu=1$.

The leading term in (14), and therefore in $\Delta \mathcal{E}$ in (9), is of order $|B|$. In a wide class of models the sign of the coefficient turns out to be negative so that the energy density is lowered by $B \neq 0$.

Since the integrand in (14) behaves like $\ln p^{2}$ (or $1 / p^{4}$ ) for small (or large) $p$, the integral converges. In particular, one can Wick-rotate the $p_{0}$ integral. Hence in terms of $\mathbf{p}=\left(\vec{p}, p_{3}=\right.$ $\left.-i p_{0}\right)$

$$
\Delta \mathcal{E}^{(1)}-\Delta \mathcal{E}^{(2)}=\frac{1}{2} \int \frac{d^{3} \mathbf{p}}{(2 \pi)^{3}} \ln \frac{\left(1+\Pi_{0}\right)\left\{1+\frac{1}{\mathbf{p}^{2}}\left(p_{3}^{2} \Pi_{0}+\vec{p}^{2} \Pi_{2}\right)\right\}+\frac{1}{\mathbf{p}^{2}}\left(\kappa-\Pi_{1}\right)^{2}}{(B \rightarrow 0)} .
$$

It is in order to give a simple model. Suppose that a system is chirally symmetric, i.e. there are an equal number of $\eta=+$ and - fermions $\left(N_{f}^{+}=N_{f}^{-}=N_{f}\right)$, each pair having the same mass and charge. Because of (18), $\left.\Pi_{1}^{\text {tot }}(p)\right|_{B=0}=0$. If all fermions have the same charge $q_{a}=e$, and all $\eta=+(-)$ fermions have $\nu=1(\nu=0)$, the condition (8) or (23) reads

$$
\kappa=\frac{e^{2}}{2 \pi} N_{f} .
$$

In the massless fermion limit

$$
\left.\Pi_{1}^{\mathrm{tot}}(p)\right|_{B \neq 0} \sim \frac{e^{2} N_{f}}{2 \pi} \frac{2}{2+\mathbf{p}^{2} l^{2}} .
$$

$\Pi_{0}^{\text {tot }}$ and $\Pi_{2}^{\text {tot }}$ are given by $2 N_{f}$ times the formula in (24) up to $\mathrm{O}\left(B^{2}\right)$ corrections.

Hence in the argument of the logarithm in (27), the numerator is always smaller than the denominator, implying that the energy difference is negative. One finds

$$
\Delta \mathcal{E}=-\frac{N_{f} e^{2}}{2 \pi^{3}} \tan ^{-1} \frac{4}{\pi} \cdot e|B|+\mathrm{O}\left(|B|^{3 / 2}\right)
$$


The energy density is minimized at $B \neq 0$, the Lorentz invariance being spontaneously broken. The true vacuum is a Hall liquid with $\nu=1$ for $\eta=1$ fermions. If $\kappa<0$, the condition is satisfied by having $\nu=1(\nu=0)$ for $\eta=-(+)$. Although the sign of $B$ is not determined in this approximation, higher order corrections will lift the degeneracy if $\kappa \neq 0$.

As a generalization one can consider a case where $\kappa=\left(e^{2} / 2 \pi\right) N_{0}\left(N_{0}\right.$ : a positive integer $)$, $N_{f}^{+}=N_{f}^{-}=N_{f}>N_{0}$. If $N_{0}$ fermions of $\eta=+$ have $\nu=1$ and all others have $\nu=0$, the two conditions, (8) and $\Delta \mathcal{E}(B \neq 0)<0$, are satisfied.

In the case of QED, namely with $\kappa=0$, the situation is opposite. If the fermion content is chirally symmetric so that $\left.\Pi_{1}^{\text {tot }}(p)\right|_{B=0}=0$, then $\nu=1$ fermions give positive contributions of $\mathrm{O}(|B|)$ to $\Delta \mathcal{E}$. The energy is minimized at $B=0$, which is consistent with the assertion that chiral symmetry is dynamically broken. ${ }^{2,3}$ If the fermion content is not chirally symmetric, the sign of an $\mathrm{O}(|B|)$ term in $\Delta \mathcal{E}$ depends on both $\left\{\nu_{a}\right\}$ and $\left\{m_{a}\right\}$, and a detailed numerical evaluation is necessary to determine the sign.

Finally we would like to clarify the relation between the condition (23) and NambuGoldstone theorem. We first note that the location of poles of the photon propagator is determined by zeros of

$$
\operatorname{det} D^{-1}(p)=\left(p^{2}\right)^{2}\left\{\left(1+\Pi_{0}\right)\left(p^{2}+p_{0}^{2} \Pi_{0}-\vec{p}^{2} \Pi_{2}\right)-\left(\kappa-\Pi_{1}\right)^{2}\right\} .
$$

The front factor $\left(p^{2}\right)^{2}$ represents gauge degrees of freedom, while the factor in the braket determines the photon spectrum $p_{0}(\vec{p})$. The condition (23) implies that

$$
\lim _{\vec{p} \rightarrow 0} p_{0}(\vec{p})=0,
$$

i.e. a photon is a massless excitation.

On the other hand we have an identity

$$
\lim _{p \rightarrow 0} \int d^{3} x e^{i p x} \partial_{\rho}^{x}\left\langle\mathrm{~T}\left[\mathcal{M}^{0 j \rho}(x) F_{0 k}(0)\right]\right\rangle=i \epsilon^{j k}\left\langle F_{12}(0)\right\rangle .
$$

Here $\mathcal{M}^{\mu \nu \rho}=x^{\mu} T^{\nu \rho}-x^{\nu} T^{\mu \rho}$ where $T^{\mu \nu}$ is the symmetric energy-momentum tensor. $M^{0 j}=\int d^{2} x \mathcal{M}^{0 j 0}$ generates a Lorentz boost. (33) implies that a dynamically generated $B=-\left\langle F_{12}\right\rangle \neq 0$ must accompany a massless excitation which couples to $\mathcal{M}^{0 j \rho}$ and $F_{0 k}$. It is nothing but a photon. Its massless nature, (32), is guaranteed by the Nambu-Goldstone theorem associated with the spontaneous breaking of the Lorentz invariance. Consequently the relation (23) should acquire no higher order corrections. ${ }^{12}$

Under a Lorentz boost the new vacuum is transformed into a state with $B \neq 0$ and $F_{0 j} \neq 0$. The transformed state has a higher energy, being unstable against pair creation. There is no degeneracy associated with the spontaneous symmetry breaking. The Nambu-Goldstone boson is a vector, but not a scalar.

In this paper we have shown that the Lorentz invariance is spontaneously broken in a wide class of models by dynamical generation of a magnetic field. We have considered only integer filling ( $\nu=0$ or 1 ), in which case the mechanism works only for a quantized bare Chern-Simons coefficient. For a general value of the coefficient, one needs to consider fermion states of fractional filling. This, with applications to non-relativistic systems, will be discussed separately. 


\section{Acknowledgements}

This work was supported in part by the U.S. Department of Energy under contract no. DE-AC02-83ER-40105. The author would like to thank Valery Rubakov, Yannick Meurice, Koichi Yamawaki, and Eduardo Fradkin for many enlightening discussions at the early or final stage of investigation. He is grateful to the Aspen Center for Physics for its hospitality, where a part of work was done.

\section{References}

1. A.N. Redrich, Phys. Rev. Lett. 52, 18 (1984); Phys. Rev. D29, 2366 (1984); K. Ishikawa, Phys. Rev. Lett. 53, 1615 (1984); Phys. Rev. D31, 1432 (1985).

2. T. Appelquist, D. Nash, and L.C.R. Wijewardhana, Phys. Rev. Lett. 60, 2575 (1988); D. Nash, Phys. Rev. Lett. 62, 3024 (1989); T. Appelquist and D. Nash, Phys. Rev. Lett. 64, 721 (1990); R. Pisarski, Phys. Rev. D44, 1866 (1991); M.R. Pennington and D. Walsh, Phys. Lett. 253B, 313 (1991); D.C. Curtis, M.R. Pennington, and D. Walsh, Phys. Lett. 295B, 313 (1992); M.C. Diamantini, P. Sodano, and G.W. Semenoff, Phys. Rev. Lett. 70, 3848 (1993).

3. E. Dagotto, J. Kogut, and A. Kocic, Phys. Rev. Lett. 62, 1083 (1988).

4. A. Zee, Int. J. Mod. Phys. B5, 529 (1991); Mod. Phys. Lett. B5, 1339 (1991); P. Wiegmann, Topological superconductivity, Chicago preprint; E. Fradkin, (private communication).

5. S.C. Zhang, T. Hansson, and S. Kivelson, Phys. Rev. Lett. 62, 82 (1989); J. Jain, Phys. Rev. Lett. 63, 199 (1989); Phys. Rev. B40, 8079 (1989); A. Balatsky and E. Fradkin, Phys. Rev. B43, 10622 (1991); A. Lopez and E. Fradkin, Phys. Rev. B44, 5246 (1991).

6. See e.g. V.B. Berestetskii, E.M. Lifshitz, and L.P. Pitaevskii, "Quantum Electrodynamics" (Pergamon Press, 1982), section 32.

7. Computations here are parallel to those in the anyon superconductivity. We follow the notation in Y. Hosotani, Int. J. Mod. Phys. B7, 2219 (1993).

8. See e.g. A.A. Abrikosov, L.P. Gorkov, and I.E. Dzyaloshinski, "Methods of Quantum Field Theory in Statistical Physics" (Dover Publications, 1975).

9. S. Randjbar-Daemi, A. Salam, and J. Strathdee, Nucl. Phys. B340, 403 (1990); and ref. 7.

10. Useful relations are (8-10) and (8-12), with typos there corrected, in R.P. Feynman and A.R. Hibbs, "Quantum Mechanics and Path Integrals" (McGraw-Hill, 1965).

11. Non-invariant (chirally asymmetric) Pauli-Villars regularization is unnatural in the $\kappa \rightarrow 0$ limit.

12. S. Coleman and B. Hill, Phys. Lett. 159B, 184 (1985); H. So, Prog. Theoret. Phys. 74, 585 (1985); N. Imai, K. Ishikawa, T. Matsuyama, and I. Tanaka, Phys. Rev. B42, 10610 (1990). 